\title{
Case report: electrical storm during induced hypothermia in a patient with early repolarization
}

\author{
Patrick Badertscher ${ }^{1,2}$ (D) Michael Kuehne ${ }^{1,2}$, Beat Schaer ${ }^{1,2}$, Christian Sticherling ${ }^{1,2}$, Stefan Osswald ${ }^{1,2}$ \\ and Tobias Reichlin ${ }^{1,2^{*}}$
}

\begin{abstract}
Background: Population based studies showed an association of early repolarization in the electrocardiogram (ECG) and a higher rate of sudden cardiac death presumably due to ventricular fibrillation. The triggers for ventricular fibrillation in patients with early repolarization are not fully understood.

Case presentation: We describe the case of a young patient with a survived ventricular fibrillation arrest while asleep followed by multiple episodes of recurrent ventricular fibrillation. The admission ECG showed an early repolarization pattern with substantial J-point elevation in most of the ECG-leads. After initiation of a hypothermia protocol, the patient developed an electrical storm with multiple ventricular fibrillation episodes requiring multiple cardioversions. Intravenous isoproterenol infusion successfully suppressed the malignant arrhythmia.

Conclusion: Hypothermia appears proarrhythmic in patients with early repolarization and may trigger ventricular fibrillation. This knowledge is particularly important when initiating temperature management protocols in patients after a survived cardiac arrest. During the acute phase of an early repolarization associated electrical storm, isoproterenol is the most effective treatment suppressing the ventricular fibrillation-inducing premature ventricular complexes at higher heart rates.
\end{abstract}

Keywords: Early repolarization, Sudden cardiac death, Ventricular fibrillation, Electrical storm, Hypothermia, Antiarrhythmic drugs

\section{Background}

Population based studies showed an association of early repolarization (ER) in the electrocardiogram (ECG) and a higher rate of sudden cardiac death presumably due to ventricular fibrillation (VF) $[1,2]$. The triggers for VF in patients with ER are not fully understood [3]. Based on this case we discuss the possible link between hypothermia and recurrent VF episodes in the context of ER.

\section{Case presentation}

A 31-year-old man suffered an out-of-hospital cardiac arrest during the night while asleep. His girlfriend started CPR immediately. Upon arrival of the emergency

* Correspondence: tobias.reichlin@usb.ch

${ }^{1}$ Cardiovascular Research Institute Basel (CRIB), University Hospital Basel, Basel, Switzerland

${ }^{2}$ Department of Cardiology, University Hospital Basel, Petersgraben 4, 4031 Basel, $\mathrm{CH}$, Switzerland medical team, the patient was found in VF and defibrillation could restore spontaneous circulation. He had no significant past medical history and was taking no medications. Family's medical history revealed that the patient's sister had implanted a pacemaker due to sicksinus syndrome, at the age of 22 years. Plasma electrolyte levels, including potassium and calcium, were within normal ranges. Toxicological screening was negative. The ECG at presentation showed sinus rhythm and an ER pattern with substantial J-point elevation in most of the ECG-leads with notching of the terminal part of the QRS complex in the lateral leads and slurring in the inferior leads (Fig. 1). Coronary angiography revealed an anomalous origin of the left anterior descending (LAD) coronary artery arising from a separate ostium of the right sinus of Valsalva without any other concomitant congenital anomaly. In the absence of neurological 


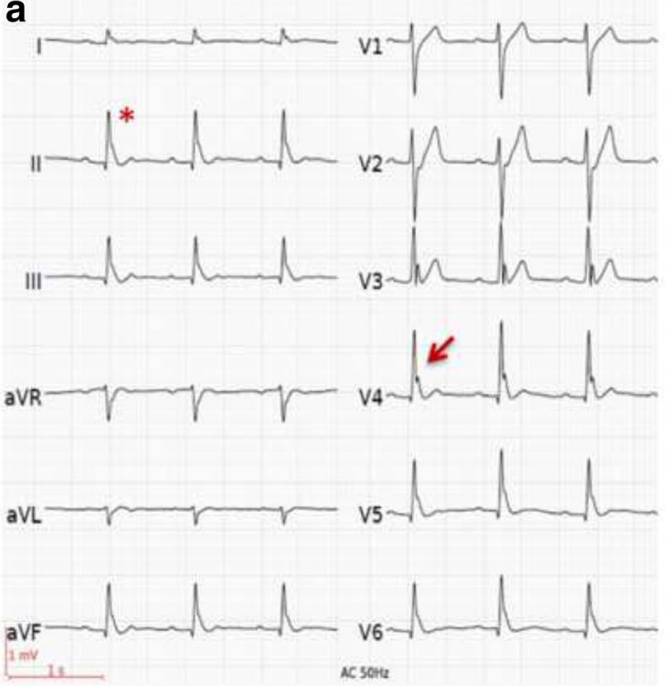

b

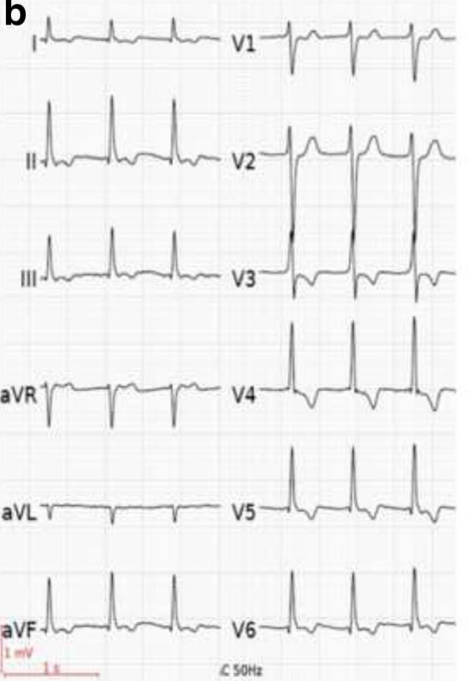

Fig. 1 ECG at presentation (a) and during isoproterenol infusion (b). Panel A shows the electrocardiogram (ECG) at presentation showing major QRS-T abnormality with substantial J-point elevation in most of the ECG-leads and notching of the terminal part of the QRS complex in the lateral leads (arrow) and slurring of the terminal part of the QRS complex in the inferior leads (*). Panel B shows near-normalization of QRS morphology during isoproterenol infusion. The notch in the lateral leads disappeared and the slur in the inferior leads was markedly attenuated. New $T$ wave inversions are present, probably attributed to cardiac memory

recovery or purposeful movements, a targeted temperature management protocol with a goal temperature of $36^{\circ} \mathrm{C}$ was started in the intensive care unit [4]. Three hours later, the patient began to experience very frequent episodes of spontaneous VF. The episodes of VF were induced by shortcoupled monomorphic premature ventricular complexes (PVC) falling into the vulnerable descending part of the Twave (Fig. 2). The PVCs showed a right bundle block morphology in V1 and a right-inferior axis in the limb leads, suggesting an origin in the left anterior fascicle. The patient was shocked for more than $10 \mathrm{VF}$ episodes, but the PVC kept reoccurring, sometimes just a few beats after cardioversion (Fig. 2b). The patient's condition was finally stabilized by isoproterenol infusion, which successfully suppressed the PVC's at higher heart rates in sinus rhythm. During the course of the isoproterenol infusion, a reduction

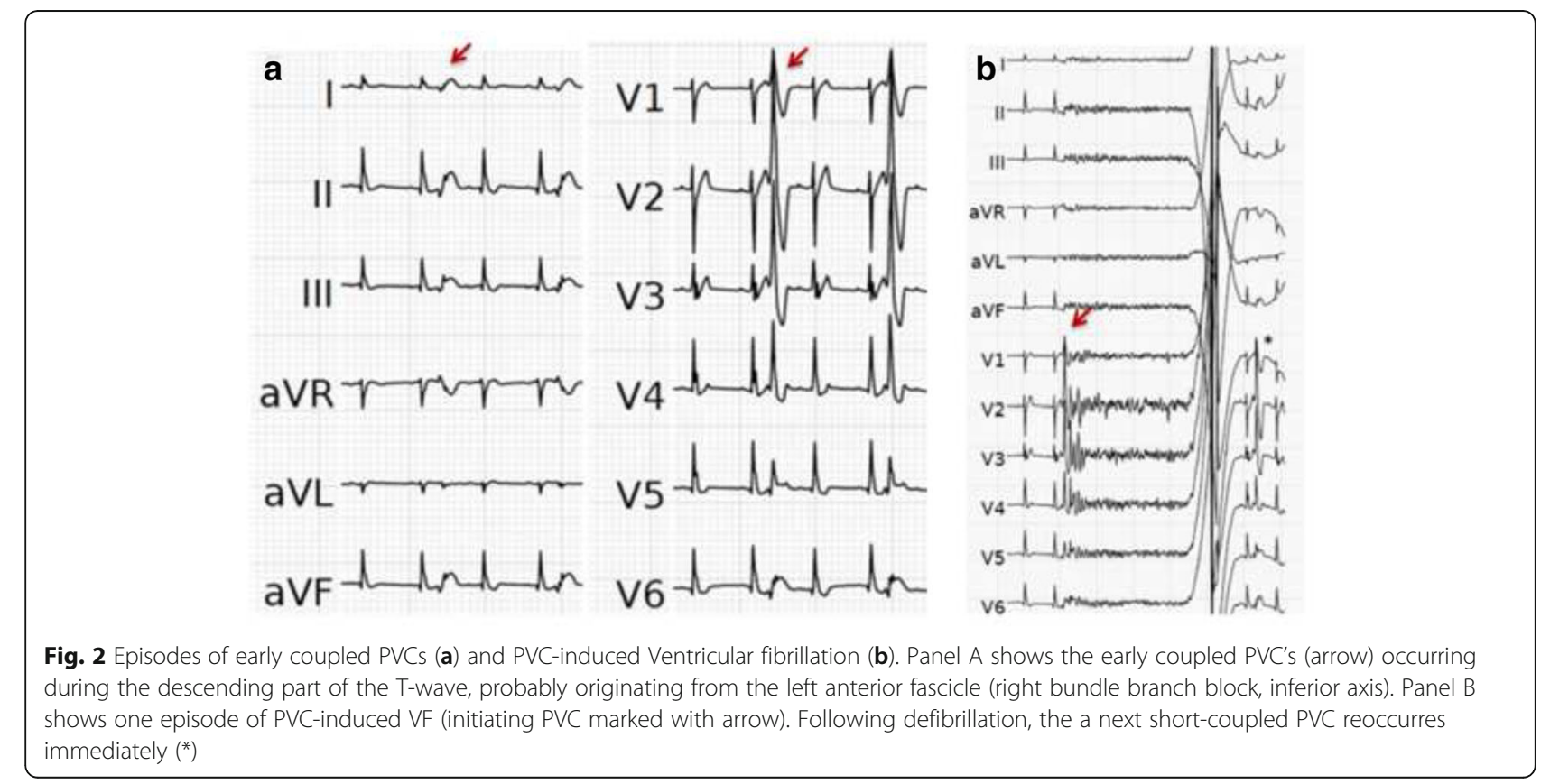


of the ER pattern on the 12-lead ECG was observed (Fig. 1b). Compared to the ECG at presentation (Fig. 1a) the notch in the lateral leads disappeared and the slur in the inferior leads was markedly attenuated. Remarkably, new $\mathrm{T}$ wave inversions were present, possibly attributed to cardiac memory. Two days later, the patient was extubated and showed a good neurologic recovery. Cardiac magnetic resonance (CMR) imaging demonstrated normal cardiac structure and function and no late gadolinium enhancement. Regarding the anomalous origin of the LAD from the opposite sinus CMR revealed an interarterial course, in which the LAD passes between the aorta and pulmonary trunk. The left circumflex (LCX) artery was located in its usual expected position at the left sinus of Valsalva (Fig. 3). A dual chamber ICD was implanted allowing for continued atrial pacing. A myocardial perfusion imaging with bicycle exercise stress testing was performed four weeks after the hospitalization and showed no signs of ischemia. The patient has not experienced any further arrhythmias during the first two months of follow-up.

\section{Discussion}

ER is common, occurring in $1 \%$ to $5 \%$ of the general population [1]. It is more common in young adults (particularly men) and athletes. The ECG pattern is defined as the presence of a J-point elevation $\geq 1 \mathrm{~mm}$ in $\geq 2$ contiguous inferior and/or lateral leads with or without STelevation, either as a QRS slurring (an abrupt change in the slope of the last deflection) or notching (a lowfrequency deflection at the end of the QRS complex) [5]. These criteria are fulfilled in our patient as illustrated in Fig. 1a. ER syndrome (ERS) is diagnosed in the presence of this ECG pattern and resuscitation of otherwise unexplained VF or polymorphic VT [5].
Historically ER was considered benign. Population based studies by Tikkanen et al. [1] then showed a higher rate of SCD among patients with ER, casecontrol studies by Haïssaguerre et al. [2] found an increased prevalence of ER in patients with idiopathic VF $(31 \%-42 \%)$ compared with healthy control subjects $(5 \%-13 \%)$. This was true for J-point elevations in the inferior leads and leads I to aVL, but not in leads V4-V6, where the frequency was similar. Importantly, subjects with ER and idiopathic VF were more likely to have sustained a cardiac arrest during sleep, which was also the case in our patient. Haïssaguerre et al. [2] noted an increase in the amplitude of ER prior to an arrhythmic period. This finding was consistent with a preliminary analysis [6] of four patients with ER and idiopathic electrical storm, in which an unique electrocardiographic signature was found. A baseline ER pattern with dramatic but transient accentuation of $J$ waves across the precordial and limb leads before the development of the electrical storm, which was precipitated by relatively short-coupled PVCs, was observed in all four patients. The same electrocardiographic signature was seen in our patient: the ER pattern was most pronounced immediately before the electrical storm, with subsequent ECG recordings after the electrical storm demonstrating a progressive decrease in J-wave height and slurring, respectively notching (Fig. 1a vs. b).

With regard to risk stratification, Antzelevitch et al [3] described three subtypes of ER with a varying risk profile: in type 1, the ER pattern is limited to the lateral precordial leads. It is typically seen in healthy male athletes and has the lowest risk of malignant arrhythmias. Type 2 shows ER in the inferior and inferolateral leads and is associated with a greater risk of malignant arrhythmias and type 3 shows ER pattern in all ECG leads and has
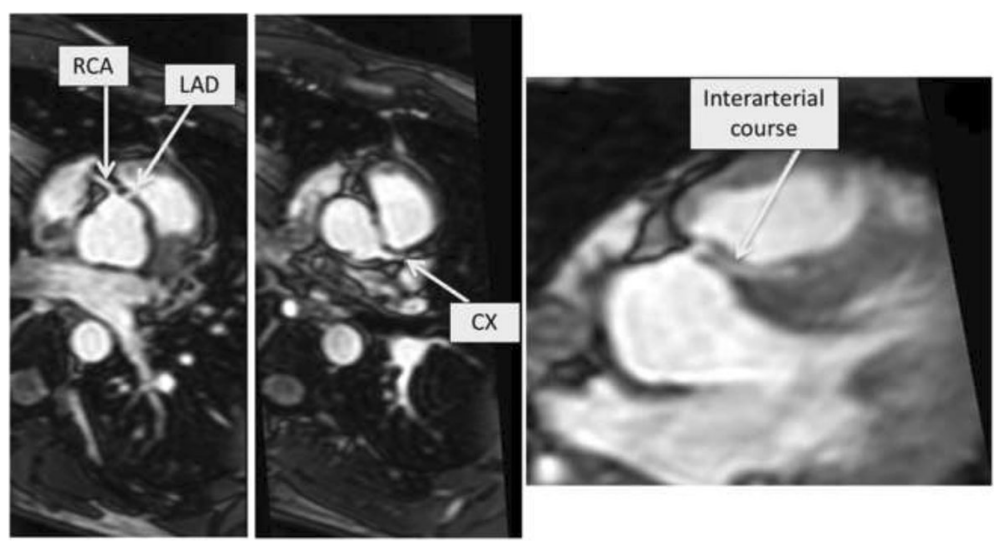

Fig. 3 Cardiovascular magnetic resonance imaging showing interaterial course of left anterior descending artery. Cardiovascular magnetic resonance imaging showing the course of the anomalous left anterior descending coronary artery (LAD) arising from a separate ostium of the right sinus of Valsalva with a potentially malignant interarterial course, in which the LAD passes between the aorta and pulmonary trunk. The left circumflex (LCX) artery is located in its usual expected position at the left sinus of Valsalva 
the highest risk of malignant arrhythmias and electrical storms. The ER pattern of our patient was most likely compatible with type 2 . In addition to the location, a Jpoint elevation of $>0.2 \mathrm{mV}$ was previously shown to be linked to a significant risk of death from cardiac arrhythmias [1]. And also distinct T-wave features have been used for the risk stratification in ER patients [7].

Different triggers leading to an electrical storm can be discussed. Recent reports point to an association between ER and the development of VF in the setting of hypothermia [8-10]. Current guidelines recommend a targeted temperature management protocol to prevent neurological damage following a cardiac arrest. In our patient, a target temperature of $36{ }^{\circ} \mathrm{C}$ was used. Electrical storm started and exacerbated after initiation of cooling and no arrhythmias occurred after it was stopped, suggesting a temporal relationship between hypothermia and increased frequency of ventricular arrhythmias in our patient. Although Brugada syndrome and ER differ in many aspects, a possible relationship to temperature is suspected in both entities, as fever appeared to be proarrhythmic in Brugada patients [11].

The finding of an LAD originating from the right sinus of Valsalva is extremely rare $(0.03 \%)$ [12]. In general, this subtype of an anomalous origination of a coronary artery from the opposite sinus (ACAOS) is associated with congenital heart disease, e.g. tetralogy of Fallot. The anatomical variant with an interarterial course of the LAD between the ascending aorta and the pulmonary trunk is considered high risk for myocardial ischemia or sudden death as well [13]. The mechanism of sudden death is believed to be due to transient occlusion of the LAD caused by an increase in blood flow through the aorta and the pulmonary artery, resulting in kinking or pinching of the artery [13]. In our case, an interarterial course was present. However, before admission, the patient was asymptomatic without any complaints during daily activity and regular exercise and the VF arrest occurred while sleeping, making ACAOS and resulting myocardial ischemia a very unlikely cause of his VF arrest. Owing to a lack of clear guideline recommendations, the management of patients with ACAOS remains fraught with uncertainty. Of note, there are no controlled studies that have evaluated the outcome of surgical repair in asymptomatic individuals. We decided that further risk stratification with myocardial perfusion imaging stress testing (MPI) was warranted. The MPI was performed four weeks after the hospitalization and showed no signs of ischemia.

Different therapeutic management options for electrical storms in ERS patients can be discussed. For the acute phase, beta-blockers, verapamil and lidocaine/ mexiletine are generally ineffective, while amiodarone may be partially effective (3/10 patients) [14]. The most effective strategy in the acute phase of electrical storms however seems the use of isoproterenol infusion or pacing at rapid heart rates to suppress the PVC's [14]. In addition, Isoproterenol is thought to be effective probably by boosting L-type calcium channel current and thus decreasing electrical gradient [3]. The efficacy of isoproterenol infusion has additionally been reported in a case report of an electrical storm occurring in a patient with Brugada syndrome [15]. For the long-term prevention of recurrent VF in ERS patients, quinidine is the drug of choice [14]. Catheter ablation for idiopathic VF targets the short coupled PVC triggers. In experienced hands, it can have a good long-term success in preventing VF recurrence [16]. However, presence of the PVC's during the procedure is required for mapping and successful ablation and recurrent VF episodes may be induced during ablation due to triggered automaticity. Therefore, quinidine is often chosen as the first line option for long-term suppression and catheter ablation used in patients with recurrent electrical storms in ERS patients. Importantly, due to the limited data with regard to sample size and follow-up duration $[14,16]$, neither successful catheter ablation nor quinidine should be considered alternatives to the implantation of an ICD for secondary prevention of SCD. In summary, ER is associated with idiopathic VF and hypothermia appears proarrhythmic in patients with ER and idiopathic VF. It remains uncertain whether additional avoidable triggers of arrhythmogenesis exist in patients with ER. Interventions shown to be effective are isoproterenol and quinidine. Further research of the genetics and mechanisms involved are required.

\section{Conclusion}

Intensive care physicians should be aware of hypothermia as a possible trigger of electrical storm in patients with ERS. This knowledge is particularly important when initiating temperature management protocols in patients after a survived cardiac arrest. During the acute phase of an electrical storm, isoproterenol is the most effective treatment suppressing the VF-inducing PVCs at higher heart rates. For the long-term management, both catheter ablation of the PVC triggers and quinidine have been used successfully to reduce or even prevent recurrent VF episodes.

\section{Abbreviations \\ CMR: Cardiac magnetic resonance; ECG: Electrocardiogram; ER: Early repolarization; ERS: Early repolarization syndrome; ICD: Implantable cardioverter-defibrillator; LAD: Left anterior descending; PVC: Premature ventricular complexes; VF: Ventricular fibrillation}

\section{Acknowledgments}

None.

Funding

No funding was received for this study. 


\section{Availability of data and materials}

The datasets used and/or analysed during the current study are available from the corresponding author on reasonable request.

\section{Authors' contributions}

PB analyzed and interpreted the data, drafted the manuscript and made critical revision of the manuscript for important intellectual content. MK made critical revision of the manuscript for important intellectual content and analyzed the data. BS cared for the patient in the inpatient and outpatient clinic, made critical revision of the manuscript for important intellectual content and analyzed the data. CS cared for the patient in the inpatient and outpatient clinic, made critical revision of the manuscript for important intellectual content, analyzed the data and handled supervision. SO made critical revision of the manuscript for important intellectual content. TR cared for the patient in the inpatient and outpatient clinic, analyzed and interpreted the data and made critical revision of the manuscript for important intellectual content. All authors read and approved the final manuscript.

\section{Ethics approval and consent to participate}

Written informed consent was obtained from the patient for participation in this case report in line with the local institutional review board of the University Hospital Basel. A copy of the written consent is available for review by the Editor-in-Chief of this journal.

\section{Consent for publication}

Written informed consent was obtained from the patient for publication of this case report and any accompanying images. A copy of the written consent is available for review by the Editor-in-Chief of this journal.

\section{Competing interests}

The authors declare that they have no competing interests.

\section{Publisher's Note}

Springer Nature remains neutral with regard to jurisdictional claims in published maps and institutional affiliations.

Received: 4 July 2017 Accepted: 8 November 2017

Published online: 15 November 2017

\section{References}

1. Tikkanen JT, Anttonen O, Junttila MJ, et al. Long-term outcome associated with early repolarization on electrocardiography. N Engl J Med. 2009; 361(26):2529-37. https://doi.org/10.1056/NEJMoa0907589.

2. Haïssaguerre M, Derval N, Sacher F, et al. Sudden cardiac arrest associated with early repolarization. N Engl J Med. 2008;358(19):2016-23. doi:10.1056/ NEJMoa071968.

3. Antzelevitch C, Yan G-X. J Wave Syndromes. Heart Rhythm. 2010;7(4):549-58. doi:10.1016/j.hrthm.2009.12.006.

4. Nielsen N, Wetterslev J, Cronberg T, et al. Targeted temperature management at $33^{\circ} \mathrm{C}$ versus $36^{\circ} \mathrm{C}$ after cardiac arrest. N Engl J Med. 2013; 369(23):2197-206. doi:10.1056/NEJMoa1310519.

5. MacFarlane PW, Antzelevitch C, Haissaguerre M, et al. The early repolarization pattern. A consensus paper J Am Coll Cardiol. 2015 doi:10.1016/j.jacc.2015.05.033.

6. Nam G-B, Kim Y-H, Antzelevitch C. Augmentation of J waves and electrical storms in patients with early repolarization. N Engl J Med. 2008;8(35819): 2078-9.

7. Roten $L$, Derval N, Maury $P$, et al. Benign vs malignant inferolateral early repolarization: focus on the T wave. Heart Rhythm. 2016;13(4):894-902. doi:10.1016/j.hrthm.2015.11.020.

8. Yamaki M, Sato N, Imanishi R, Sakai H, Kawamura Y, Hasebe N. Low room temperature can trigger ventricular fibrillation in J wave syndromes. Heart Case Reports. 2016;2(4):347-50. doi: 10.1016/j.hrcr.2016.04.003.

9. Federman NJ, Mechulan A, Klein GJ, Krahn AD. Ventricular fibrillation induced by spontaneous hypothermia in a patient with early repolarization syndrome. J Cardiovasc Electrophysiol. 2013;24(5):586-8. doi:10.1111/jce. 12030.

10. Bastiaenen R, Hedley PL, Christiansen M, Behr ER. Therapeutic hypothermia and ventricular fibrillation storm in early repolarization syndrome. Heart Rhythm. 2010;7(6):832-4. doi:10.1016/j.hrthm.2010.02.037.
11. Keller D, Rougier J, Kucera J, et al. Brugada syndrome and fever: genetic and molecular characterization of patients carrying mutations. Cardiovasc Res. 2005;67(3):510-9. doi:10.1016/j.cardiores.2005.03.024.

12. Yamanaka $\mathrm{O}$, Hobbs RE. Catheterization curriculum coronary artery anomalies in 126,595 patients undergoing coronary arteriography. Catheter Cardiovasc Diagn. 1990;21:28-40.

13. Basso C, Maron BJ, Corrado D, Thiene G. Clinical profile of congenital coronary artery anomalies with origin from the wrong aortic sinus leading to sudden death in young competitive athletes. J Am Coll Cardiol. 2000; 35(6):1493-501. doi:10.1016/S0735-1097(00)00566-0.

14. Haïssaguerre $M$, Sacher $F$, Nogami $A$, et al. Characteristics of recurrent ventricular fibrillation associated with inferolateral early repolarization. Role of Drug Therapy J Am Coll Cardiol. 2009;53(7):612-9. doi:10.1016/j.jacc.2008. 10.044 .

15. Maury P, Couderc P, Delay M, Boveda S, Brugada J. Electrical storm in Brugada syndrome successfully treated using isoprenaline. Europace. 2004; 6(2):130-3. doi:10.1016/j.eupc.2003.11.009.

16. Knecht S, Sacher F, Wright M, et al. Long-term follow-up of idiopathic ventricular fibrillation ablation. A Multicenter Study J Am Coll Cardiol. 2009; 54(6):522-8. doi:10.1016/j.jacc.2009.03.065.

\section{Submit your next manuscript to BioMed Central and we will help you at every step:}

- We accept pre-submission inquiries

- Our selector tool helps you to find the most relevant journal

- We provide round the clock customer support

- Convenient online submission

- Thorough peer review

- Inclusion in PubMed and all major indexing services

- Maximum visibility for your research

Submit your manuscript at www.biomedcentral.com/submit
C Biomed Central 